\title{
Feasibility of Diagnosing Initial Orthostatic Hypotension Using a Continuous Blood Pressure Device in Geriatric Rehabilitation Inpatients: RESORT
}

\author{
Jennifer Tran ${ }^{a}$ Arjen Mol ${ }^{b}$ Rebecca K. Iselia Wen Kwang Lim ${ }^{a}$ \\ Carel G.M. Meskers ${ }^{\mathrm{b}}$ Andrea B. Maier ${ }^{\mathrm{a}, \mathrm{c}, \mathrm{d}}$
}

\begin{abstract}
${ }^{a}$ Department of Medicine and Aged Care, the Royal Melbourne Hospital, The University of Melbourne, Parkville, VIC, Australia; ${ }^{b}$ Department of Human Movement Sciences, Amsterdam Movement Sciences, Vrije Universiteit Amsterdam, Amsterdam, The Netherlands; ' ${ }^{\text {Department }}$ of Rehabilitation Medicine, Amsterdam UMC, Vrije Universiteit, Amsterdam Movement Sciences, Amsterdam, The Netherlands; ${ }^{d}$ Healthy Longevity Translational Research Program, Yong Loo Lin School of Medicine, National University of Singapore, Centre for Healthy Longevity, National University Health System, Singapore, Singapore
\end{abstract}

\section{Keywords}

Aged · Blood pressure monitors - Initial orthostatic

hypotension · Orthostatic · Geriatrics

\begin{abstract}
Background: Initial orthostatic hypotension $(\mathrm{IOH})$ is highly prevalent in older adults and may interfere with the ability to regain function after acute hospitalization. $\mathrm{IOH}$ assessment requires a non-invasive, beat-to-beat continuous blood pressure device, which is not widely used in geriatric rehabilitation. Our aim was to test the feasibility of diagnosing $\mathrm{IOH}$ using a continuous blood pressure device in geriatric rehabilitation inpatients. Methods: Geriatric rehabilitation inpatients of the REStORing Health of Acutely Unwell AdulTs (RESORT) cohort admitted to a tertiary hospital were randomly selected to undergo continuous blood pressure monitoring (Finapres) for $5 \mathrm{~min}$ in the supine position and $3 \mathrm{~min}$ of standing or sitting when unable to stand. Interventions to warm hands and adjusting the cuff pressure sizes were attempted if no signal was obtained or an error message occurred. Results: Of 37 randomly selected inpatients, 29 \{55.2\% female; mean age 82.8 (standard deviation [SD]) 6.6 years\} agreed to the continuous blood pressure measure-
\end{abstract}

karger@karger.com www.karger.com/ger

Karger!"

GOPEN ACCESS
C 2022 The Author(s).

Published by S. Karger AG, Basel

This is an Open Access article licensed under the Creative Commons Attribution-NonCommercial-4.0 International License (CC BY-NC) (http://www.karger.com/Services/OpenAccessLicense), applicable to the online version of the article only. Usage and distribution for commercial purposes requires written permission. ment. Successful measurements were achieved in 20 out of 29 inpatients, two after hand warming. Patients with unsuccessful measurements were likely to be older (mean age 87.2 [SD] 4.4 years, $p=0.03$ ), have cerebrovascular disease ( $p=$ $0.006)$, lower body mass index $(p=0.012)$, and a lower short physical performance battery score $(p=0.039)$. Eight out of 20 patients had $\mathrm{IOH}$. Conclusion: The number of unsuccessful continuous blood pressure measurements was high in a population with high $\mathrm{IOH}$ prevalence despite multiple interventions to establish a signal. Future research should focus on improving the efficiency of continuous blood pressure devices in hospitalized patients with unsuccessful signals.

(c) 2022 The Author(s).

Published by S. Karger AG, Basel

\section{Introduction}

Initial orthostatic hypotension ( $\mathrm{IOH})$ is a clinical syndrome defined as a transient decrease of $\geq 40 \mathrm{~mm} \mathrm{Hg}$ systolic blood pressure (SBP) and/or $\geq 20 \mathrm{~mm} \mathrm{Hg}$ diastolic blood pressure (DBP) within $15 \mathrm{~s}$ of active standing [1]. The prevalence of $\mathrm{IOH}$ is $27.8 \%$ in the older general populations and $35.2 \%$ in geriatric outpatients [2] with a high
Correspondence to:

Andrea B. Maier, a.b.maier@vu.nl 
prevalence of orthostatic intolerance symptoms in older adults, with $\mathrm{IOH}$ ranging between 24 and 100\% [3]. Unlike classical orthostatic hypotension $(\mathrm{COH})$ which can be detected using a conventional sphygmomanometer [4], the diagnosis of $\mathrm{IOH}$ requires non-invasive, beat-tobeat, continuous blood pressure monitoring to evaluate blood pressure changes occurring within $60 \mathrm{~s}$ after active standing [4] or passive tilting [1]. These measurements rely on the volume-clamp method [5]. Currently, the clinical use of continuous blood pressure devices in older adults is limited due to its high cost and poor applicability [6]. However, the assessment of haemodynamic responses to postural change is important in older adults due to the association of $\mathrm{IOH}$ with hospitalizations, injurious falls, frailty, and mortality $[7,8]$.

Limited studies have assessed the feasibility of using continuous blood pressure devices like Finapres NOVA to measure IOH in hospitalized older adults [2] due to the more complex application of continuous blood pressure devices than the use of sphygmomanometers [6]. Use of continuous blood pressure devices may be particularly limited by blood circulation impairments in geriatric patients with acute and chronic diseases and subsequent high medication use. Because of this, determining $\mathrm{IOH}$ in geriatric rehabilitation patients poses a clinical challenge. However, implementing continuous blood pressure devices in routine clinical care for older adults may help to identify $\mathrm{IOH}$ sooner and aid clinicians to minimize the negative outcomes associated with $\mathrm{IOH}$ [9].

The aims of this study were to assess the feasibility of diagnosing $\mathrm{IOH}$ using continuous blood pressure measurement in geriatric rehabilitation inpatients by establishing the number of successful IOH measurements defined as the presence of a signal with measurable waveforms throughout the last $60 \mathrm{~s}$ before postural change and during the $180 \mathrm{~s}$ of active stand or sit. Patient characteristics of successful and unsuccessful measurements were compared.

\section{Methods}

\section{Study Population and Setting}

The REStORing health of acutely unwell adulTs (RESORT) is an ongoing prospective, longitudinal, observational cohort of subacute geriatric rehabilitation patients at the Royal Melbourne Hospital (Victoria, Australia), assessing their physical, cognitive, and functional status. In a random sample of patients admitted between February 14, 2020, and March 13, 2020, continuous blood pressure measurements were added to the study protocol. Written informed consent was obtained by the patient themselves or a nominated proxy. Patients were excluded if they were receiving palliative care, transferred to acute care prior to consenting to the study, or incapable of providing informed consent due to delirium or severe dementia without a nominated proxy.

\section{Patient Characteristics}

Patients were assessed within $48 \mathrm{~h}$ of admission to the geriatric rehabilitation wards by physicians, nurses, physiotherapists, occupational therapists, and dieticians. Information on age, sex, smoking status (current or past smoking), morbidity, and medication were extracted from medical charts. Comorbidity was assessed using the Charlson Comorbidity Index [10] and Cumulative Illness Rating Scale [11]. Cardiovascular disease included any past history of angina, myocardial infarction, cardiac failure, valvular pathology, atrial fibrillation, or other arrhythmias [12]. Cognitive impairment was assessed by physicians and defined as being present if it was recognized on either the Charlson comorbidity index or Cumulative Illness Rating Scale; mild cognitive impairment or dementia mentioned as a diagnosis in the discharge summary; or standardized Mini-Mental State Examination [13] score of $<24$ points, Rowland Universal Dementia Assessment Scale (RUDAS) [14] score of $<23$ points, and Montreal Cognitive Assessment [15] score of $<26$ points. Fall history was defined by selfreported falls in the last 12 months. Physical functioning was assessed by the physiotherapist using the short physical performance battery assessment [16] and functional ambulation classification [17] and by the occupational therapist using the Katz Index of ADL [18] and the Lawton and Brody's instrumental activities of daily living (IADL) scale [19]. The frailty status was assessed using the 9-point Clinical Frailty Scale (CFS) (1 is considered fit and 9 extremely frail [20]). Nutritional status was assessed by the body mass index [21] and malnutrition screening tool [22].

\section{Continuous Blood Pressure Measurement - Experimental}

Protocol

Continuous blood pressure measurements were performed by a trained staff member using a non-invasive, beat-to-beat blood pressure monitoring device (Finapres NOVA; Finapres Medical Systems, Amsterdam, The Netherlands). A finger cuff was applied to the patient's non-dominant middle phalanx of the third digit and an oscillometric pressure cuff on the ipsilateral upper arm. The patient was asked to maintain the monitored arm across their chest at the heart level, where the height sensor was placed to calibrate and correct for any remaining differences in height between the finger sensor and heart. Patients remained in the supine position for $5 \mathrm{~min}$ in silence with the Finometer Physiocal function (i.e., the device calibration mechanism to adjust cuff inflation pressure based on the photoplethysmogram) enabled. Manual markings were made on the blood pressure recording once the patient attained a stable supine position and again at the start of transition from supine to standing or sitting position. A cuff size guide ranging from small, medium, or large was used to measure the patient's middle phalanx to determine which cuff size was appropriate. Cuff sizes were readjusted if a cuff error signal was present. If there was no or temporary signal, warming of the hands was performed, firstly, attempted by wearing double-latex gloves on the hand with the finger cuff for $30 \mathrm{~min}$, and if unsuccessful, again attempted with a warm wet towel for another $30 \mathrm{~min}$. Just before standing, Finometer Physiocal function was immediately disabled to prevent measurement discontinuities during the measurement. Simultaneously, patients were asked to stand up from the supine position 


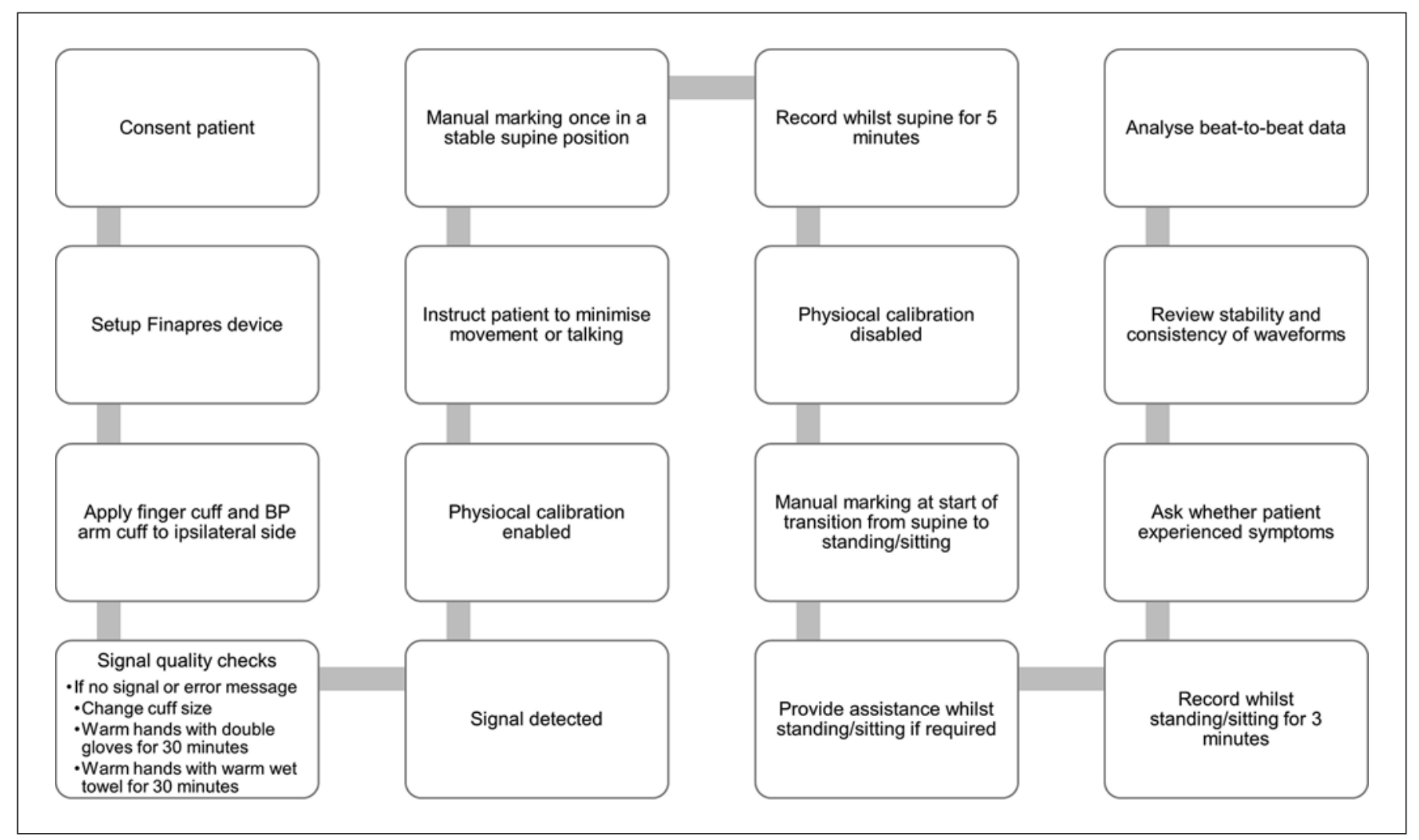

Fig. 1. Schematic timeline of the experimental protocol.

as quickly as they could and timed with a stopwatch. If patients were not able to stand up, they were asked to assume a sitting position instead. Physical assistance including holding the patient's free arm for support was provided if standing or sitting could not be achieved independently. After postural change, patients remained standing for $3 \mathrm{~min}$ in silence, with the monitored arm maintained at the heart level. Immediately following the blood pressure measurement, patients were asked to report whether they experienced symptoms of light-headedness, blurred vision, dizziness, or nausea during postural change. A successful measurement was defined as the presence of a signal with measurable waveforms (i.e., no errors or measurement discontinuities) throughout the last $60 \mathrm{~s}$ before postural change and the $180 \mathrm{~min}$ of active stand or sit. An unsuccessful measurement was defined as the absence of a signal, poor waveforms, or recurrent error messages during the continuous blood pressure measurement. A schematic timeline of the experimental protocol is demonstrated in Figure 1.

\section{Blood Pressure Data Analysis}

Beat-to-beat data of blood pressure and heart rate (HR) were exported to MATLAB (version R2012b; MathWorks, Natick, MA, USA) and filtered using a 5-s moving average filter to attenuate artefacts. Blood pressure parameters obtained to assess $\mathrm{IOH}$ included supine SBP and supine DBP, defined as the average blood pressure in the last $60 \mathrm{~s}$ before postural change from the supine position to standing. The relative blood pressure decrease was determined by subtracting the lowest blood pressure at 3 time intervals defined from the start of standing $(0-15,15-60$, and 60-180 s) from the supine blood pressure.

\section{Definitions of $\mathrm{IOH}$ and $\mathrm{COH}$}

IOH was defined as a decrease of $\geq 40 \mathrm{~mm} \mathrm{Hg} \mathrm{SBP}$ and/or $\geq 20$ mm Hg DBP within $15 \mathrm{~s}$ of active standing or sitting, and $\mathrm{COH}$ was defined as a sustained decrease of $\geq 20 \mathrm{~mm} \mathrm{Hg} \mathrm{SBP}$ and/or $\geq 10$ $\mathrm{mm} \mathrm{Hg}$ DBP within 3 min of active standing or sitting [1].

\section{Statistical Analysis}

Statistical analyses were performed using Statistical Package for the Social Sciences (IBM SPSS Advanced Statistics 24.0; IBM Corp, Armonk, NY, USA). Categorical variables are presented as a numerical value $(n)$ with percentages (\%), continuous variables with a normal distribution were reported as means and standard deviations (SDs), and skewed distribution (non-Gaussian) data are reported as medians with interquartile ranges. Characteristics between patients with successful and unsuccessful measurements were compared using an unpaired sample $t$ test for continuous data, Mann-Whitney $U$ test for skewed distribution data or the $\chi^{2}$ test for categorical data. A $p$ value of less than 0.05 was considered statistically significant. 


\section{Results}

A total of 29 out of 37 randomly selected geriatric rehabilitation inpatients underwent continuous blood pressure measurements. Three patients refused, one patient was unable to provide consent due to a non-English speaking background, 3 due to severe delirium, and 1 had a fistula and lymph node removal on both arms. A flow chart detailing the final cohort is shown in Figure 2. Table 1 shows the participant characteristics for the 29 included patients (mean age was 82.8 years (SD 6.6), 55.2\% female) who underwent continuous blood pressure monitoring.

\section{Successful versus Unsuccessful Measurements}

During the first attempt of measurements using the continuous blood pressure device, 18 out of 29 patients had a successful measurement. Of the 11 patients who had a poor signal, 9 patients underwent 2 hand warming interventions due to no signal or an error message (i.e., beat detection timeout or LED current problem), which was effective in 2 patients. The remaining 2 patients had a recurrent error message relating to cuff pressure problems. Of the 20 patients with a successful measurement, 15 patients were able to perform an active stand, and 5 patients were able to perform sitting. The total time to complete a successful blood pressure measurement in the first attempt took less than $15 \mathrm{~min}$. The total time to complete a continuous blood pressure measurement in the 9 patients who required multiple attempts due to a poor signal was longer than an hour, with each hand warming intervention lasting $30 \mathrm{~min}$ each.

\section{Differences between the Successful and Unsuccessful \\ Measurement Group}

Patient's characteristics stratified by a successful ( $n=$ $20)$ or unsuccessful $(n=9)$ measurement are given in Table 2. Patients with unsuccessful measurements were older $(p=0.03)$, more likely to have cerebrovascular disease ( $p=0.006)$, and had a lower short physical performance battery score $(p=0.039)$ and lower body mass index $(p=$ 0.012 ). There were no statistically significant differences in cognitive impairment or frailty between inpatients with a successful or unsuccessful measurement.

\section{Blood Pressure Characteristics of Inpatients with the Successful Measurement Group}

Table 3 presents the blood pressure characteristics of inpatients with successful measurement group. One participant was excluded in the HR analyses due to poor
Table 1. Characteristics of geriatric rehabilitation inpatients at admission

\begin{tabular}{lll}
\hline Characteristics & $N$ & Value \\
\hline Sociodemographic & & \\
$\quad$ Age, mean \pm SD, years & 29 & $83.2 \pm 6.6$ \\
$\quad$ Female sex, $n$ & 29 & 16 \\
$\quad$ Current or past smoker, yes, $n$ & 27 & 10 \\
Medical history and medication & & \\
$\quad$ CCI, score (0-37) & 29 & $2[1-3]$ \\
CIRS, score (0-56) & 29 & $11[8-12]$ \\
Cardiovascular disease, yes, $n$ & 29 & 19 \\
$\quad$ Type-2 diabetes mellitus, yes, $n$ & 29 & 8 \\
$\quad$ Cerebrovascular disease, yes, $n$ & 29 & 3 \\
Parkinsonism or Parkinson's disease, & & \\
$\quad$ yes, $n$ & 29 & 3 \\
$\quad$ Medications number & 29 & $10[7.25-14.75]$ \\
$\quad$ Cognitive impairment, yes, $n$ & 29 & 18 \\
Physical functioning & & \\
Falls in previous 12 months, yes, $n$ & 29 & 22 \\
SPPB, score (0-12) & 29 & $3[1-6]$ \\
FAC, score (0-5) & 28 & $3[2-3.75]$ \\
$\quad$ KADL, median (IQR), score (0-6) & 29 & $2[1-4]$ \\
$\quad$ IADL, median (IQR), score (0-8) & 29 & $1[1-2.5]$ \\
Frailty & & \\
CFS, score (0-9) & 29 & $5[4-6]$ \\
Nutritional status & & \\
BMI, mean \pm SD, kg/m ${ }^{2}$ & 29 & $28.6 \pm 7.6$ \\
MST, score (0-5) & 28 & $0[0-2]$ \\
\hline
\end{tabular}

All values are presented as mean (IQR) unless otherwise stated. IADL, Lawton and Brody's instrumental activities of daily living; $\mathrm{KADL}$, Katz Index of activities of daily living; $\mathrm{CCl}$, Charlson comorbidity index; SPPB, short physical performance battery; FAC, functional ambulation classification; BMI, body mass index; MST, malnutrition screening tool; IQR, interquartile range; CIRS, Cumulative IIIness Rating Scale.

quality of data. Mean baseline supine SBP and DBP were $141.2 \mathrm{~mm} \mathrm{Hg}$ (SD 25.5) and $75.0 \mathrm{~mm} \mathrm{Hg}$ (SD 14.8), respectively, and HR was $74.0 \mathrm{bpm}$ (SD 11.0). Within the first $15 \mathrm{~s}$, the mean SBP magnitude drop was $36.0 \mathrm{~mm} \mathrm{Hg}$ (SD 28.7, range 88.1), DBP magnitude drop was $18.3 \mathrm{~mm}$ $\mathrm{Hg}$ (SD 14.6, range 51.6), and HR increase was $28.3 \mathrm{bpm}$ (22.9-43.4, range 121.8). Representative beat-to-beat waveforms of the blood pressure and HR responses to postural change are shown in Figure 3. IOH was present in 8 out of 20 patients. Of those with IOH, 6 performed active stand, and 2 performed sitting. $\mathrm{COH}$ was prevalent in 13 out of 20 patients. Four of the 8 patients with $\mathrm{IOH}$ and 7 of the 21 patients without $\mathrm{IOH}$ reported at least one or more symptoms of orthostatic intolerance (light-headedness, blurred vision, dizziness, or nausea). 


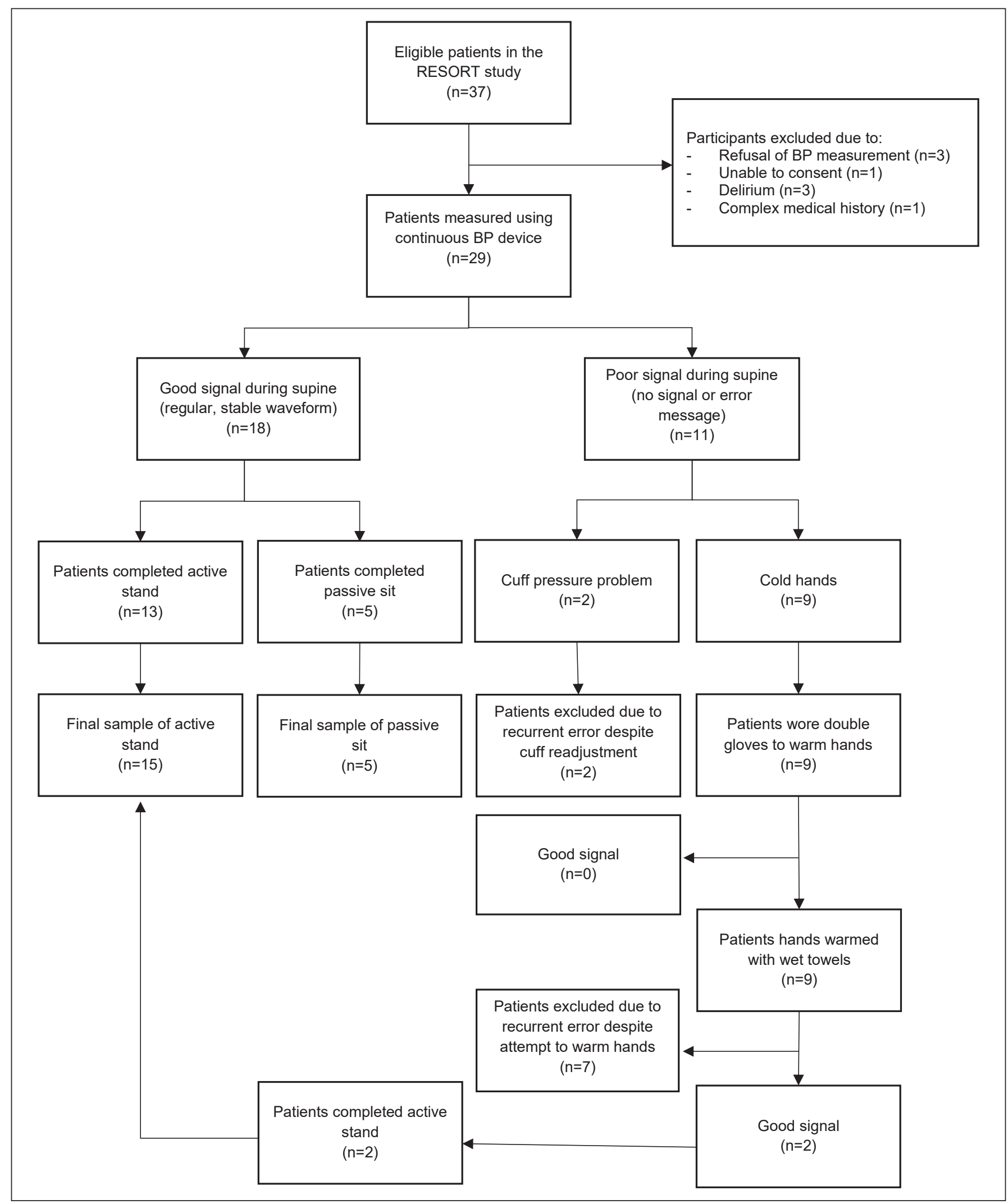

Fig. 2. Flow chart detailing final subsample analysed. 
Table 2. Comparison of patient characteristics stratified by successful and unsuccessful measurements

\begin{tabular}{|c|c|c|c|}
\hline Characteristics & $\begin{array}{l}\text { Successful } \\
(n=20)\end{array}$ & $\begin{array}{l}\text { Unsuccessful } \\
(n=9)\end{array}$ & $p$ value \\
\hline \multicolumn{4}{|l|}{ Sociodemographic } \\
\hline Age, mean $\pm S D$, years & $81.5 \pm 6.8$ & $87.2 \pm 4.4$ & $0.030 *$ \\
\hline Female sex, $n$ & 12 & 4 & 0.436 \\
\hline Current or past smoker, yes, $n$ & 8 & 2 & 0.351 \\
\hline \multicolumn{4}{|l|}{ Medical history and medication } \\
\hline $\mathrm{CCl}$, score $(0-37)$ & $6[4.8-7]$ & $6[5-7]$ & 0.943 \\
\hline CIRS, score $(0-56)$ & $11[9-12]$ & $11[6-12]$ & 0.585 \\
\hline Cardiovascular disease, yes, $n$ & 5 & 2 & 0.872 \\
\hline Type-2 diabetes mellitus, yes, $n$ & 7 & 1 & 0.183 \\
\hline Cerebrovascular disease, yes, $n$ & 0 & 3 & $0.006^{*}$ \\
\hline Parkinsonism or Parkinson's disease, yes, $n$ & 1 & 2 & 0.159 \\
\hline Medication number & $10[8-15]$ & $7[5-10]$ & 0.127 \\
\hline Cognitive impairment, yes, $n$ & 11 & 7 & 0.242 \\
\hline \multicolumn{4}{|l|}{ Physical functioning } \\
\hline Falls in previous 12 months, yes, $n$ & 16 & 6 & 0.438 \\
\hline SPPB, score (0-12) & $4[2.8-7]$ & $1[0-2]$ & $0.039 *$ \\
\hline FAC, score $(0-5)$ & $3[2.5-4]$ & $3[1-3]$ & 0.128 \\
\hline KADL, median (IQR), score (0-6) & $2[1-4]$ & $1[0-2]$ & 0.184 \\
\hline IADL, median (IQR), score (0-8) & $1[1-2]$ & $2[0-3]$ & 1.000 \\
\hline \multicolumn{4}{|l|}{ Frailty } \\
\hline CFS, score (0-9) & $5[4-6]$ & $6[4-6]$ & 0.379 \\
\hline \multicolumn{4}{|l|}{ Nutritional status } \\
\hline $\mathrm{BMI}$, mean $\pm \mathrm{SD}, \mathrm{kg} / \mathrm{m}^{2}$ & $30.9 \pm 7.7$ & $23.5 \pm 4.6$ & $0.012 *$ \\
\hline MST, score $(0-5)$ & $0[0-2]$ & $0[0-2]$ & 0.549 \\
\hline
\end{tabular}

All values are presented as mean (IQR) unless otherwise stated. IADL, Lawton and Brody's Instrumental Activities of Daily Living; KADL, Katz Index of Activities of Daily Living; CCI, Charlson comorbidity index; SPPB, short physical performance battery; FAC, functional ambulation classification; BMI, body mass index; MST, malnutrition screening tool; IQR, interquartile range; CIRS, Cumulative Illness Rating Scale. * Statistically significant $p<0.05$.

\section{Discussion}

Almost a third of geriatric rehabilitation inpatients had an unsuccessful continuous blood pressure measurement during active standing, impairing the diagnosis of $\mathrm{IOH}$ and potentially causing underestimation of $\mathrm{IOH}$ prevalence. The poor success rate of continuous blood pressure measurements is unsurprising. High vessel stiffness and endothelial dysfunction [23] are common physiological ageing processes that are prevalent in the geriatric population [24] and can compromise cerebral vasculature [25] and peripheral circulation [26]. Older patients with poor peripheral circulation due to conditions like cold fingers, arthritic fingers, Parkinson's disease (causing rigidity and/ excessive tremor), peripheral vascular disease, or systemic vasculitis [27] can make it challenging to record a reliable continuous blood pressure measurement [28] as it relies on pulsatile unloading of the finger arterial walls [27].
Feasibility of continuous blood pressure measurements in the clinical setting poses additional challenges. The total time to complete a blood pressure measurement using a continuous blood pressure device should take less than $15 \mathrm{~min}$ [29]. This includes patient setup and instruction, calibration of finger arterial pressure, $5 \mathrm{~min}$ in the supine position, $3 \mathrm{~min}$ of active standing or passive sitting, and symptom reporting. A large proportion of our patients had initially a poor signal which meant that the total time to complete a blood pressure measurement extended to over an hour with the addition of the 2 hand warming interventions. Considering the low success rates and the significant time investment required for each blood pressure measurement, implementing routine $\mathrm{IOH}$ screening using Finapres may prove challenging in a regular clinical setting.

There are several strategies to optimize the quality of blood pressure recordings, including warming the hand with double-examination gloves and a warm wet towel 
Table 3. Blood pressure values and orthostatic intolerance symptoms for inpatients with a successful continuous measurement

\begin{tabular}{|c|c|}
\hline Blood pressure characteristics & $\begin{array}{l}\text { Value } \\
(n=20)\end{array}$ \\
\hline Supine SBP, mean (SD), mm Hg & $141.2(25.5)$ \\
\hline Supine DBP, mean (SD), $\mathrm{mm} \mathrm{Hg}$ & $75.0(14.8)$ \\
\hline Supine HR, mean (SD), bpm & $74.0(11.0)$ \\
\hline \multicolumn{2}{|l|}{ Prevalence of $\mathrm{IOH}$ and $\mathrm{COH}$} \\
\hline $\mathrm{IOH}, n$ & 8 \\
\hline $\mathrm{IOH}$ in an active stand $(n=15), n$ & 6 \\
\hline $\mathrm{IOH}$ in passive sitting $(n=5), n$ & 2 \\
\hline $\mathrm{IOH}(n=8)+\mathrm{COH}, n$ & 6 \\
\hline $\mathrm{COH}, n$ & 12 \\
\hline $\mathrm{COH}$ in an active stand $(n=15), n$ & 8 \\
\hline $\mathrm{COH}$ in passive sitting $(n=5), n$ & 4 \\
\hline $\mathrm{COH}(n=12)+\mathrm{IOH}, n$ & 6 \\
\hline \multicolumn{2}{|c|}{ Orthostatic blood pressure and HR responses in patients with $\mathrm{IOH}$} \\
\hline SBP drop magnitude, $0-15 \mathrm{~s}$, mean (SD), $\mathrm{mm} \mathrm{Hg}$ & $65.9(15.8)$ \\
\hline DBP drop magnitude, $0-15 \mathrm{~s}$, mean (SD), $\mathrm{mm} \mathrm{Hg}$ & $28.3(11.7)$ \\
\hline HR increase $0-15 \mathrm{~s}$ in $1 / \mathrm{s}$, median (IQR) & $28.2[26.3-55.3]$ \\
\hline \multicolumn{2}{|c|}{ Orthostatic blood pressure and HR responses in patients without $\mathrm{IOH}$} \\
\hline SBP drop magnitude, $0-15 \mathrm{~s}$, mean (SD), $\mathrm{mm} \mathrm{Hg}$ & $16.0(13.2)$ \\
\hline DBP drop magnitude, $0-15 \mathrm{~s}$, mean (SD), $\mathrm{mm} \mathrm{Hg}$ & $11.7(12.6)$ \\
\hline HR increase $0-15 \mathrm{~s}$ in $1 / \mathrm{s}$, median (IQR]) & $28.3[19.7-40.7]$ \\
\hline \multicolumn{2}{|c|}{ Symptoms of orthostatic intolerance in patients with $\mathrm{IOH}(n=8)$} \\
\hline Positive symptom(s), $n$ & 4 \\
\hline \multicolumn{2}{|c|}{ Symptoms of orthostatic intolerance in patients without $\mathrm{IOH}(n=12)$} \\
\hline Positive symptoms(s), $n$ & 7 \\
\hline \multicolumn{2}{|c|}{$\begin{array}{l}\qquad \mathrm{IOH}=\text { a drop of } \geq 40 \mathrm{~mm} \mathrm{Hg} \mathrm{SBP} \text { and/or } \geq 20 \mathrm{~mm} \mathrm{Hg} \mathrm{DBP} \text {, within } 15 \mathrm{~s} \text { after active standing } \\
\text { or passive sitting. } \mathrm{COH}=\text { a decrease of } \geq 20 \mathrm{~mm} \mathrm{Hg} \mathrm{SBP} \text { and } \geq 10 \mathrm{~mm} \mathrm{Hg} \text { during } 15-180 \mathrm{~s} \\
\text { after active standing or sitting. SBP, drop magnitude = difference between baseline SBP and } \\
\text { the lowest measured SBP value in the standing/sitting intervals at } 0-15,15-60 \text {, and } 60-180 \\
\text { s. Positive symptom(s) is defined as reporting one or more of the following: light-headedness, } \\
\text { blurred vision, dizziness, nausea, or pain. }\end{array}$} \\
\hline
\end{tabular}

[29]. Although successful in a few patients, this method did not lead to successful measurements in all patients. One study noted improvements in blood pressure measurements when hands were warmed with warm water or a glove filled with warm water [30]. However, this strategy would not be particularly suitable in older patients who are at high risk of falls, especially if unsupervised. Other strategies to consider may include creating multiple cuff sizes to appropriately fit fingers that were too short, overly thin, or arthritic, increasing room temperature to promote cutaneous vasodilation [31], reducing anxiety to reduce peripheral vasoconstriction [32], or attempting to measure continuous blood pressure at an alternative anatomical site. During the blood pressure measurement itself, minimizing external pressures applied to the finger pressure cuff (i.e., pushing off against the bed or supporting oneself during postural change) can reduce significant artefacts during postural change. A possible solution to decreasing movement artefact or contaminating the blood pressure signal is by supporting the measured arm with a sling [29].

Identifying IOH regardless of the presence of symptoms $[33,34]$ in geriatric rehabilitation inpatients is clinically important due to the associated high risks of falls, frailty, and syncope $[33,35-38]$. This association is due to age-related changes like decreased skeletal muscle strength and cardiorespiratory conditioning which can impair restoration of systemic blood pressure $[35,39,40]$. Currently, continuous blood pressure devices are not readily available in clinical practice due to the high costs and poor applicability [6], leading to underdiagnosis of $\mathrm{IOH}$. Possible strategies to combat $\mathrm{IOH}$, if detected, include rationalizing medications (particularly antihypertensives and psychoactive drugs $[41,42])$, standing up 


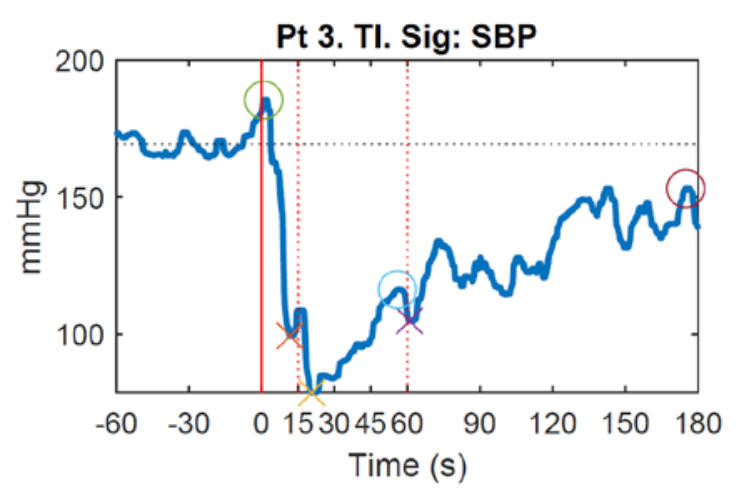

Pt 3. TI. Sig: DBP

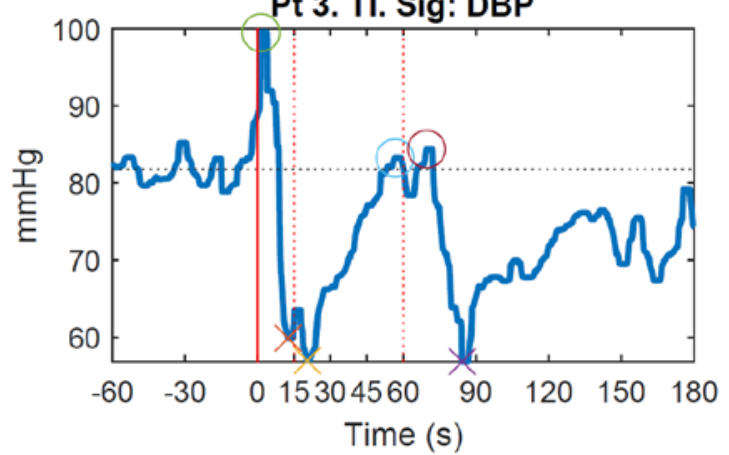

Pt 3. TI. Sig: HR

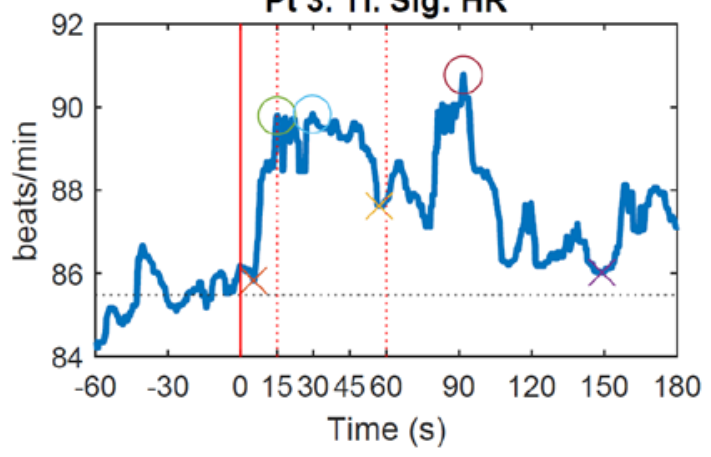

Fig. 3. SBP, DBP, and $\mathrm{HR}$ response to active standing in a patient with IOH. The waveforms show a 5-s moving average filter. Time 0 : start of postural change. The solid red lines indicate the start and end of postural change. The dotted red line indicates the time $15 \mathrm{~s}$ after the end of postural change. The horizontal dotted line indicates the mean value during the $60 \mathrm{~s}$ before postural change. The orange, yellow, and purple crosses indicate the minimum value for intervals between $0-15,15-60$, and $60-180 \mathrm{~s}$ after the end of postural change, respectively. Green, blue, and red circles indicate the maximum value in intervals between $0-15,15-60$, and $15-180 \mathrm{~s}$ after the end of postural change, respectively.

slowly for prolonged activation of skeletal muscle pump [9], contracting lower body muscles to prevent venous pooling [42], and salt supplementation [43]. If IOH is left undetected and thus untreated, this may consequently lead to poorer clinical outcomes [6]. This study highlights the importance of improving the reliability of continuous blood pressure devices during active stand in geriatric rehabilitation patients to detect $\mathrm{IOH}$ [44].

\section{Strengths and Limitations}

This is the first study to assess the feasibility of using continuous blood pressure devices to determine initial $\mathrm{OH}$ in hospitalized older adults. The strength of this study was the use of a well-defined cohort of older patients admitted to geriatric rehabilitation, providing a clinically relevant study population. There are a few limitations worth noting in our study. No restrictions on hydration status, oral intake, diurnal variation of BP measurement, and medications were placed which may contribute to some measurement variability. One major limitation includes the relatively small sample size which may impact statistical power and validity of this study, possibly overestimating the rate of unsuccessful measurements reported. However, a previous study of similar sample size noted significantly underestimated baroreflex sensitivity with the use of non-invasive blood pressure measurements [45] and may be in keeping with the high unsuccessful rates in our study. Nevertheless, further studies with a larger sample size are recommended to determine the success rates of utilizing noninvasive continuous blood pressure devices to determine $\mathrm{IOH}$ in hospitalized older adults with a focus on optimizing the quality of beat-to-beat blood pressure recordings.

\section{Conclusion}

The proportion of unsuccessful blood pressure measurements using a continuous blood pressure device was high in geriatric rehabilitation inpatients, who are frequently affected by $\mathrm{IOH}$. This highlights that diagnosing $\mathrm{IOH}$ is challenging in this population in a regular clinical setting. Future research should focus on improving the efficiency of continuous blood pressure devices in hospitalized patients with unsuccessful signals.

\section{Acknowledgments}

The authors thank the multidisciplinary team members of the Royal Melbourne Hospital, Royal Park Campus, involved in the RESORT cohort for their clinical work and the @AgeMelbourne team for their role in the data collection. 


\section{Statement of Ethics}

The study was approved by Melbourne Health Human Research Ethics Committee (no. 2017.085) and follows national and international ethical guidelines according to the Helsinki Declaration, the National Statement on Ethical Conduct in Human Research (2007), and the Guidelines for Good Clinical Research Practice. Written informed consent was obtained by the patient themselves or a nominated proxy.

\section{Conflict of Interest Statement}

The authors have no conflicts of interest to declare.

\section{Funding Sources}

The research was funded by an unrestricted grant of the University of Melbourne received by Prof. Andrea B. Maier and the Medical Research Future Fund (MRFF) provided by the Melbourne Academic Centre for Health (MACH).

\section{Author Contributions}

Jennifer Tran contributed to conceptualization, methodology, formal analysis, investigation, writing - original draft, and visualization. Arjen Mol contributed to software, validation, and writing - review and editing. Rebecca $\mathrm{K}$. Iseli contributed to supervision, methodology, and writing - review and editing. Wen Kwang Lim contributed to supervision, methodology, and writing - review and editing. Carel G.M. Meskers contributed to supervision, methodology, and writing - review and editing. Andrea B. Maier contributed to conceptualization, methodology, validation, writing - review and editing, supervision, project administration, and funding acquisition.

\section{Data Availability Statement}

All data generated or analysed during this study are included in this article. Further enquiries can be directed to the corresponding author.

\section{References}

1 Freeman R, Wieling W, Axelrod FB, Benditt DG, Benarroch E, Biaggioni I, et al. Consensus statement on the definition of orthostatic hypotension, neurally mediated syncope and the postural tachycardia syndrome. Clin Auton Res. 2011;21(2):69-72.

2 Tran J, Hillebrand SL, Meskers CGM, Iseli RK, Maier AB. Prevalence of initial orthostatic hypotension in older adults: a systematic review and meta-analysis. Age Ageing. 2021; 50(5):1520-8.

3 Christopoulos EM, Tran J, Hillebrand SL, Lange PW, Iseli RK, Meskers CGM, et al. Initial orthostatic hypotension and orthostatic intolerance symptom prevalence in older adults: a systematic review. Int J Cardiol Hypertens. 2021;8:100071.

4 van Wijnen VK, Finucane C, Harms MPM, Nolan H, Freeman RL, Westerhof BE, et al. Noninvasive beat-to-beat finger arterial pressure monitoring during orthostasis: a comprehensive review of normal and abnormal responses at different ages. J Int Med. 2017; 282(6):468-83.

5 Martina JR, Westerhof BE, Van Goudoever J, De Jonge N, Van Lieshout JJ, Lahpor JR, et al. Noninvasive blood pressure measurement by the Nexfin monitor during reduced arterial pulsatility: a feasibility study. ASAIO J. 2010; 56(3):221-7.

6 de Bruine ES, Reijnierse EM, Trappenburg MC, Pasma JH, de Vries OJ, Meskers CGM, et al. Diminished dynamic physical performance is associated with orthostatic hypotension in geriatric outpatients. J Geriatr Phys Ther. 2019;42(3):E28-34.
7 Lagro J, Schoon Y, Heerts I, Meel-van den Abeelen ASS, Schalk B, Wieling W, et al. Impaired systolic blood pressure recovery directly after standing predicts mortality in older falls clinic patients. J Gerontol A Biol Sci Med Sci. 2014;69(4):471-8.

8 Romero-Ortuno R, Cogan L, Foran T, Kenny RA, Fan CW. Continuous noninvasive orthostatic blood pressure measurements and their relationship with orthostatic intolerance, falls, and frailty in older people. J Am Geriatr Soc. 2011;59(4):655-65.

9 De Bruine ES, Reijnierse EM, Trappenburg MC, Pasma JH, De Vries OJ, Meskers CGM, et al. Standing up slowly antagonises initial blood pressure decrease in older adults with orthostatic hypotension. Gerontology. 2017; 63(2):137-43

10 Charlson ME, Pompei P, Ales KL, MacKenzie CR. A new method of classifying prognostic comorbidity in longitudinal studies: development and validation. J Chronic Dis. 1987; 40(5):373-83.

11 Miller MD, Paradis CF, Houck PR, Mazumdar S, Stack JA, Rifai AH, et al. Rating chronic medical illness burden in geropsychiatric practice and research: application of the $\mathrm{cu}$ mulative illness rating scale. Psychiatry Res. 1992;41(3):237-48.

12 Hudon C, Fortin M, Soubhi H. Abbreviated guidelines for scoring the cumulative illness rating scale (CIRS) in family practice. J Clin Epidemiol. 2007;60(2):212.
13 Folstein MF, Folstein SE, McHugh PR. "Minimental state." A practical method for grading the cognitive state of patients for the clinician. J Psychiatr Res. 1975;12(3):189-98.

14 Storey JE, Rowland JT, Basic D, Conforti DA, Dickson HG. The rowland universal dementia assessment scale (RUDAS): a multicultural cognitive assessment scale. Int Psychogeriatr. 2004;16(1):13-31.

15 Nasreddine ZS, Phillips NA, Bédirian V, Charbonneau S, Whitehead V, Collin I, et al. The montreal cognitive assessment, MoCA: a brief screening tool for mild cognitive impairment. J Am Geriatr Soc. 2005;53(4):695-9.

16 Guralnik JM, Simonsick EM, Ferrucci L, Glynn RJ, Berkman LF, Blazer DG, et al. A Short physical performance battery assessing lower extremity function: association with self-reported disability and prediction of mortality and nursing home admission. Gerontol. 1994;49(2):M85-M94.

17 Viosca E, Martínez JL, Almagro PL, Gracia A, González C. Proposal and validation of a new functional ambulation classification scale for clinical use. Arch Phys Med Rehabil. 2005; 86(6):1234-8.

18 Katz S, Ford AB, Moskowitz RW, Jackson BA, Jaffe MW. Studies of illness in the aged: the index of ADL: a standardized measure of biological and psychosocial function. JAMA. 1963;185(12):914-9.

19 Lawton MP, Brody EM. Assessment of older people: self-maintaining and instrumental activities of daily living. Gerontologist. 1969; 9(3):179-86. 
20 Rockwood K, Song X, MacKnight C, Bergman H, Hogan DB, McDowell I, et al. A global clinical measure of fitness and frailty in elderly people. CMAJ. 2005;173(5):489-95.

21 Cederholm T, Bosaeus I, Barazzoni R, Bauer J, Van Gossum A, Klek S, et al. Diagnostic criteria for malnutrition: an ESPEN consensus statement. Clin Nutr. 2015;34(3):335-40.

22 Cederholm T, Jensen GL, Correia M, Gonzalez MC, Fukushima R, Higashiguchi T, et al. GLIM criteria for the diagnosis of malnutrition: a consensus report from the global clinical nutrition community. J Cachexia Sarcopenia Muscle. 2019;10(1):207-17.

23 Dai X, Hummel SL, Salazar JB, Taffet GE, Zieman S, Schwartz JB. Cardiovascular physiology in the older adults. J Geriatr Cardiol. 2015;12(3):196-201.

24 Mol A, Reijnierse EM, Trappenburg MC, Van Wezel RJA, Maier AB, Meskers CGM. Rapid systolic blood pressure changes after standing up associate with impaired physical performance in geriatric outpatients. J Am Heart Assoc. 2018;7(21):e010060.

25 Ziegler MG. Atherosclerosis and blood pressure variability. Hypertension. 2018;71(3): 403-5.

26 Miller AP, Huff CM, Roubin GS. Vascular disease in the older adult. J Geriatr Cardiol. 2016;13(9):727-32.

27 Imholz BP, Wieling W, van Montfrans GA, Wesseling KH. Fifteen years experience with finger arterial pressure monitoring: assessment of the technology. Cardiovasc Res. 1998; 38(3):605-16.

28 FMS, Finapres Medical Systems BV. Finometer user guide. Arnheim, The Netherlands: Arnheim; 2002.

29 Finucane C, van Wijnen VK, Fan CW, Soraghan C, Byrne L, Westerhof BE, et al. A prac- tical guide to active stand testing and analysis using continuous beat-to-beat non-invasive blood pressure monitoring. Clin Auton Res. 2019;29(4):427-41.

30 Edwards Lifesciences BMEYE. Nexfin HD operator's manual. Amsterdam: Edwards Lifesciences BMEYE; 2008.

31 Johnson JM, Kellogg DL Jr. Local thermal control of the human cutaneous circulation. J Appl Physiol. 2010;109(4):1229-38.

$32 \mathrm{Kim} \mathrm{JH}$, Almuwaqqat Z, Hammadah M, Liu C, Ko YA, Lima B, et al. Peripheral Vasoconstriction during mental stress and adverse cardiovascular outcomes in patients with coronary artery disease. Circ Res. 2019;125(10): 874-83.

33 Saedon NI, Zainal-Abidin I, Chee KH, Khor HM, Tan KM, Kamaruzzaman SK, et al. Postural blood pressure electrocardiographic changes are associated with falls in older people. Clin Auton Res. 2016;26(1):41-8.

34 Freeman R, Illigens BMW, Lapusca R, Campagnolo M, Abuzinadah AR, Bonyhay I, et al. Symptom recognition is impaired in patients with orthostatic hypotension. Hypertension. 2020;75(5):1325-32

35 Romero-Ortuno R, Cogan L, Foran T, Kenny RA, Fan CW. Continuous noninvasive orthostatic blood pressure measurements and their relationship with orthostatic intolerance, falls, and frailty in older people. J Am Geriatr Soc. 2011;59(4):655-65.

36 van Twist DJL, Dinh T, Bouwmans EME, Kroon AA. Initial orthostatic hypotension among patients with unexplained syncope: an overlooked diagnosis? Int J Cardiol. 2018;271: 269-73.

37 van Wijnen VK, Harms MP, Go-Schon IK, Westerhof BE, Krediet CT, Stewart J, et al. Initial orthostatic hypotension in teenagers and young adults. Clin Auton Res. 2016;26(6): 441-9.

38 Shaw BH, Borrel D, Sabbaghan K, Kum C, Yang Y, Robinovitch SN, et al. Relationships between orthostatic hypotension, frailty, falling and mortality in elderly care home residents. BMC Geriatr. 2019;19(1):80.

39 Gupta V, Lipsitz LA. Orthostatic hypotension in the elderly: diagnosis and treatment. Am J Med. 2007;120(10):841-7.

40 McJunkin B, Rose B, Amin O, Shah N, Sharma S, Modi S, et al. Detecting initial orthostatic hypotension: a novel approach. J Am Soc Hypertens. 2015;9(5):365-9.

41 Canney M, O'Connell MD, Murphy CM, O'Leary N, Little MA, O'Seaghdha CM, et al. Single agent antihypertensive therapy and orthostatic blood pressure behaviour in older adults using beat-to-beat measurements: the Irish longitudinal study on ageing. PLoS One. 2016;11(1):e0146156.

42 Rivasi G, Rafanelli M, Mossello E, Brignole M, Ungar A. Drug-related orthostatic hypotension: beyond anti-hypertensive medications. Drugs Aging. 2020;37(10):725-38.

43 El-Sayed H, Hainsworth R. Salt supplement increases plasma volume and orthostatic tolerance in patients with unexplained syncope. Heart. 1996;75(2):134-40.

44 Finucane C, Savva GM, Kenny RA. Reliability of orthostatic beat-to-beat blood pressure tests: implications for population and clinical studies. Clin Auton Res. 2017;27(1) 31-9.

45 Maestri R, Pinna GD, Robbi E, Capomolla S, La Rovere MT. Noninvasive measurement of blood pressure variability: accuracy of the finometer monitor and comparison with the finapres device. Physiol Meas. 2005;26(6): 1125-36. 